\title{
Quantum at Scale
}

\author{
Quantum technology can only fulfill its promise when it reaches a large \\ scale and proves its value to society.
}

By Joseph S. Broz, Irfan Siddiqi, and Darío Gil

This piece is the third in a three-part series. Find out more about this series in this introductory article, Ingredients for a Quantum Future.

"The future is already here-it's just not evenly distributed." These words, attributed to cyberpunk author William Gibson [1], describe to some degree the emerging quantum industry, where the technology, and the knowledge to use and appreciate it, is currently in the hands of relatively few early pioneers. But as the innovation leader and technology strategist Nicholas Davis noted in his 2016 article for the World Economic Forum [2], it is essential for an emerging technology to provably contribute to economic growth and social well-being, and to do that, the technology must be scalable. All members of society must be able to either directly access the technology or the value that

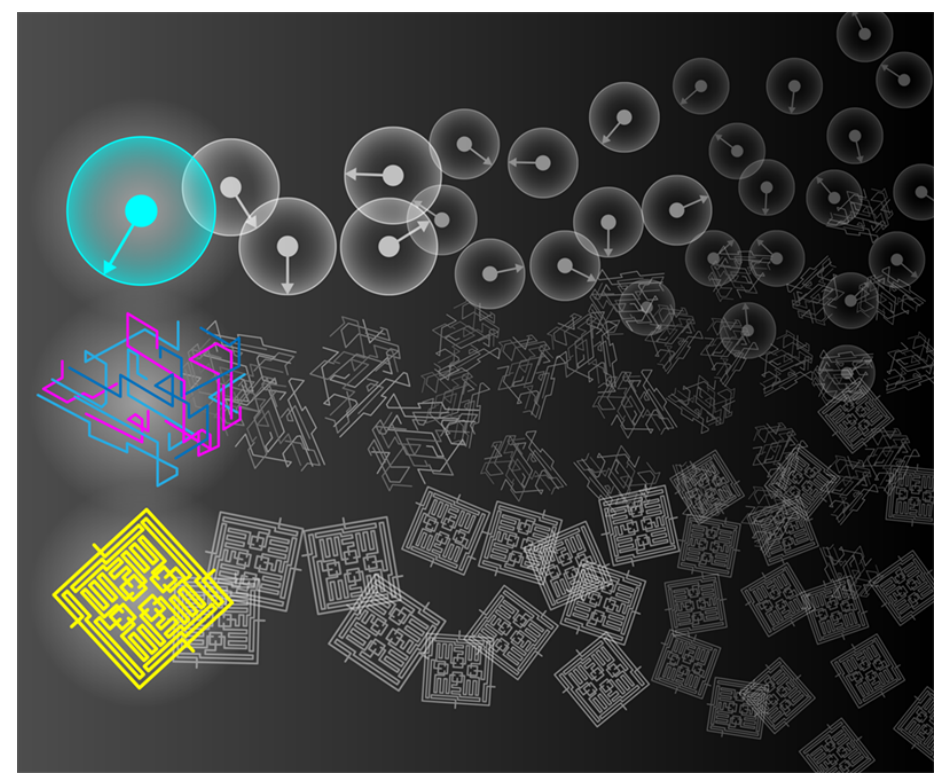

Credit: APS/Alan Stonebraker can derived from it, and at an affordable cost. In our two earlier pieces, "A Quantum Culture Shift" and "Quantum for All," we outlined the pathways to accelerating quantum R\&D and the need to democratize quantum literacy. We'll now address the issue of scale by taking a look at its main drivers.

"Scaling quantum" starts with innovation efficiency and the ability to manufacture the necessary components. Large gains in this direction would come from shifting the burden of quantum-device engineering from research laboratories and prototype developers to the manufacturing sector and the supply chain. To make collaboration easier, prototyping faster, and commercialization feasible, the quantum industry will need a reliable supply of enabling components, such as highly stable, small, low-cost lasers at exotic wavelengths; more efficient cryostats; and superconducting cables. These fit-for-use components should be readily available in standard formats and with rigorous performance specifications. Manufacturers will need to be able to rely on a stable supply of high-volume, low-cost components.

Another important element in scalability is access to universal cloud-based quantum computing resources. This access "democratizes quantum" by opening quantum computation to the broadest array of potential end users, and it speeds up innovation by giving more people the opportunity to explore new and diverse quantum computing applications. To succeed, this effort must place quantum resources into the hands of nontraditional users, outside the confines of experimental quantum physics. To this end, industry has launched programs that encourage the participation of new quantum developers and that provide them with the tools and training required to exploit quantum resources. Similar policies are forming within government-supported research. For wide utilization, quantum software will also have to be user-friendly, interoperable with 
many types of quantum hardware, and easy to access. Assistance on the latter will hopefully come from community-created repositories for quantum algorithms that are available through open-access "hubs."

Just a decade ago, only a few dozen research groups could code in quantum and perform multiqubit experiments. But that number grew to a few thousand just a week after the first online quantum computing platform launched in 2016 (the IBM Quantum Experience), which gave everyone free access to quantum processors through the cloud. Four years later, the number of programmers experimenting with quantum circuits-the sequences of instructions for manipulating data and making a quantum computer work-is in the hundreds of thousands. And in the near future, millions of software developers in the IT mainstream will build on that effort by integrating the myriad of new quantum circuits into their programs. In turn, these software innovations will touch the lives of billions of people.

Scalability will also rest on developing an "ecosystem" of stakeholders and on giving them the right education and training. Initiatives like the US Quantum Economic Development Consortium (QED-C) - a public-private consortium of around 100 US corporations, national labs, and universities and one of the most tangible outgrowths of the U.S. National Quantum Initiative Act to date-is illustrative of the desire of institutions to engage with this high-potential technology. Private sector initiatives like the IBM Quantum Network also have well over 100 institutions as members, from startups to some of the largest corporations in the world. The US Department of Energy has also recently announced the formation of five major quantum centers, complementing recent investments by the National Science Foundation and leveraging sustained investment from the Department of Defense.

It is thrilling to witness the emergence of a new quantum industry and, relatedly, an exciting time to be a researcher, entrepreneur, or student-either beginning their studies or returning back to school-with an ever-increasing set of resources available to be engaged. We encourage learning by immersion into the subject, be it in the classroom, at the stock exchange, or on the factory floor. Growth in the field will require a partnership between all stakeholders. We have good evidence that the seeds of growth and scale have already been planted, and a background in quantum will serve the tech-savvy denizens of the coming generations well.

Disclaimer: Dr. Siddiqi's views are his own and do not necessarily represent the views of the U.S. Department of Energy or the United States Government.

Joseph S. Broz: Quantum Economic Development Consortium (QED-C), Arlington, VA, USA

Irfan Siddiqi: University of California, Berkeley, and Lawrence Berkeley National Laboratory, CA, USA

Darío Gil: IBM Research, Yorktown Heights, NY, USA

\section{REFERENCES}

1. Attributed to William Gibson, cf: https://quoteinvestigator.com /2012/01/24/future-has-arrived/.

2. What is the fourth industrial revolution? Nicholas Davis, $2016 \mathrm{ht}$ tps://www.weforum.org/agenda/2016/01/what-is-the-fourth-in dustrial-revolution/. 